\title{
Laser based synchrotron radiation
}

\author{
Kim Ta Phuoc, ${ }^{\text {a) }}$ Fréderic Burgy, Jean-Philippe Rousseau, Victor Malka, and \\ Antoine Rousse \\ Laboratoire d'Optique Appliquée, ENSTA, CNRS UMR7639, Ecole Polytechnique, Chemin de la Hunière, \\ F-91761 Palaiseau, France \\ Rahul Shah and Donald Umstadter \\ Center for Ultrafast Optical Science, University of Michigan, 1006 Bonisteel Blvd, Ann Arbor 48109, \\ Michigan \\ Alexander Pukhov and Sergei Kiselev \\ Insitut fur Theoretische Physik I, Heinrich-Heine-Universitat, Duesseldorf, 40225 Dusseldorf, Germany
}

(Received 30 July 2004; accepted 8 November 2004; published online 7 January 2005)

\begin{abstract}
Beams of $\mathrm{x}$ rays in the kiloelectronvolt energy range have been produced from laser-matter interaction. Here, energetic electrons are accelerated by a laser wakefield, and experience betatron oscillations in an ion channel formed in the wake of the intense femtosecond laser pulse. Experiments using a $50 \mathrm{TW}$ laser (30 fs duration) are described, as well as comparisons with numerical simulations. These results pave the way of a new generation of radiation in the x-ray spectral range, with a high collimation and an ultrafast pulse duration, produced by the use of compact laser system. (C) 2005 American Institute of Physics. [DOI: 10.1063/1.1842755]
\end{abstract}

\section{INTRODUCTION}

Secondary x-ray radiation sources from laser-produced plasmas have undergone an important development during the last decade. Interest in them is mainly due to the short pulse duration they can provide (femtosecond time scale), as well as the compactness of the laser systems required as compared with the conventional large scale installations such as synchrotrons. High-order harmonics from solid or gas targets, extreme uv (xuv)-ray lasers and $K \alpha$ x-ray sources have been shown to produce ultrashort pulses in the xuv or x-ray spectral range. However, beams of $x$-ray radiation could not be produced until very recently. ${ }^{1}$ High-order harmonics of the fundamental laser light and xuv lasers can generate collimated radiation but only down to wavelengths of about 10 nm. ${ }^{2}$ At shorter wavelength, $K \alpha \mathrm{x}$-ray sources ${ }^{3}$ can provide subnanometers radiation. They are presently the unique plasma based x-ray sources usable for applications in ultrafast $\mathrm{x}$-ray science. ${ }^{4}$ However, their monochromaticity and full divergency (over $4 \pi$ solid angle) severely limit the applications that could be undertaken. At first, only sample showing high structural periodicity (crystals) can be studied. Information on transient atomic structures is then limited because very few Bragg peaks can be followed simultaneously during a time resolved experiment. Furthermore, complex materials cannot be investigated due to the finite size and small efficiency of the $\mathrm{x}$-ray optics used to collect the radiation. This leads to a dramatically reduced $x$-ray flux onto the sample $\left(5 \times 10^{4}\right.$ photons to be compared to the $10^{9}$ photons emitted at the source). To extend ultrafast $\mathrm{x}$-ray techniques to more complex applications (to biological and chemical applications), a brighter x-ray source, produced as a collimated beam and with a broadband spectrum is needed. Here we

\footnotetext{
${ }^{a)}$ Author to whom correspondence should be addressed. Electronic mail: kim.taphuoc@ensta.fr
}

demonstrate a novel concept that combines these features.

Recently, it was demonstrated that the large electrostatic fields generated in a laser produced plasma can be efficiently manipulated to generate and accelerate electron beams to energies comparable to that of linear accelerators (few hundreds of $\mathrm{MeV}$ ). In addition they have the unique property to be ultrafast (femtosecond). This mechanism, called forced laser wakefield, has been observed experimentally ${ }^{5}$ and from three-dimensional (3D) particle-in-cell (PIC) simulations. 5,6 Ultrafast synchrotron-type radiation can subsequently be produced by wiggling the laser-produced electron beam, outside of the plasma, in a periodic electromagnetic structure. However, for specific interaction parameters, the plasma generating the electron bunch can in fact play itself the role of wiggler. In this interaction regime, as the intense and femtosecond laser propagates in the underdense plasma (fully ionized helium gas), the ponderomotive force pushes plasma electrons away from the high intensity regions, leaving an ion channel almost free of background electrons in the wake of the laser. ${ }^{7-9}$ As the relativistic electrons propagate in the ion channel (Fig. 1), they experience a radial restoring force due to the transverse electrostatic field generated by the space charge separation with the ions. They undergo oscillations - called betatron oscillations - and, as in a conventional synchrotron, a collimated beam of synchrotron radiation originates from this relativistic and oscillatory motion. $^{7,8,10}$

Following our recent publication, ${ }^{1}$ here we show in detail how femtosecond and kiloelectronvolt (keV) synchrotron radiation is produced by this fully laser-based concept. First, a simple model is used to describe the betatron motion of a single electron and the synchrotron radiation it emits. Then, a 3D PIC code is used to simulate the full laser plasma interaction and to calculate the produced $\mathrm{x}$-ray radiation. Finally, 


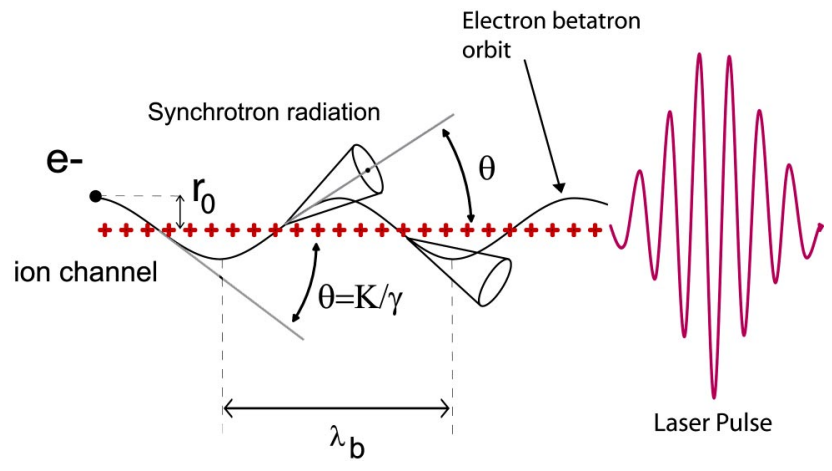

FIG. 1. (Color online). Betatron oscillation and radiation produced by a relativistic electron oscillating in an ion channel. $\theta$ is a scaled angle corresponding to the peak angular deflection of the electron. We define the parameter $K=\gamma \theta$ as the strength parameter associated to the channel. The produced synchrotron radiation is confined in a narrow cone of divergence $\theta$.

experimental results are shown and compared with the numerical simulations.

\section{X-RAY FEATURES FROM A SINGLE ELECTRON MODEL}

We consider a simple model of a single electron propagating in a uniformly charged and cylindrical ion channel in the $z$ direction (Fig. 1). The electron has an initial velocity along $z$ with an energy $\gamma_{0} m c^{2}$ (where $\gamma_{0}$ is the initial Lorentz factor) and an initial transverse position $r_{0}$ from the channel axis. As the electron propagates in the channel, it experiences the transverse restoring force $\vec{F}_{\text {res }}$ due to space charge separation with the ions. The equation of motion of the electron is then given by

$$
\frac{d \vec{p}}{d t}=\vec{F}_{\text {res }}=-m \omega_{p}^{2} \vec{r}_{\perp} / 2,
$$

where $\vec{F}_{\text {res }}$ is obtained from the Gauss law, $m$ is the electron mass, $\vec{r}_{\perp}$ the transverse position of the electron, and $\omega_{p}$ $=\left(4 \pi n_{e} e^{2} / m\right)^{1 / 2}$ the plasma frequency, with $n_{e}$ the plasma electron density and $e$ the electron charge. We assume that the restoring force is only transverse (in the direction $x$ ). The motion of the electron then lies in the $(x, z)$ plane and the axial momentum of the electron is a constant of motion. In the transverse direction, the electron oscillates at the betatron frequency $\omega_{b} \sim \omega_{p} / \sqrt{2 \gamma}$ and the motion is, at the first order, $x \sim r_{0} \cos \left(k_{b} z\right)$, where $k_{b}=\omega_{b} / c$. The trajectory obtained numerically by integrating the equation of motion using a Runge-Kutta algorithm is displayed in Fig. 2 for various pairs of parameters $\left(\gamma_{0}=60, \quad r_{0}=1 \mu \mathrm{m}\right), \quad\left(\gamma_{0}=60, r_{0}\right.$ $=0.1 \mu \mathrm{m}),\left(\gamma_{0}=20, r_{0}=0.1 \mu \mathrm{m}\right)$. The electron plasma density is $n_{e}=1 \times 10^{19} \mathrm{~cm}^{-3}$ for these simulations. The amplitude of the betatron oscillation is $r_{0}$. We can see that the frequency of the betatron oscillation decreases when the electron's initial energy increases.

The oscillatory motion of the relativistic electron in the ion channel is comparable to that of an electron oscillating in an insertion device (undulator or wiggler) of a synchrotron. Here, the ion channel acts as a wiggler-or undulator-with

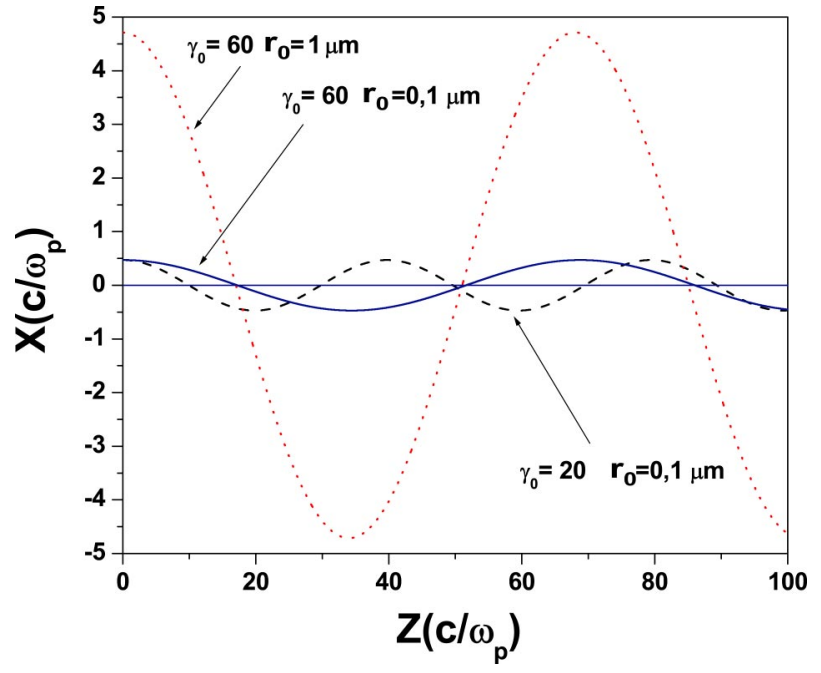

FIG. 2. Betatronic orbits of one electron for various pairs of parameters $\left(\gamma_{0}=60, r_{0}=1 \mu \mathrm{m}\right),\left(\gamma_{0}=60, r_{0}=0.1 \mu \mathrm{m}\right),\left(\gamma_{0}=20, r_{0}=0.1 \mu \mathrm{m}\right)$. The plasma density is $n_{e}=1 \times 10^{19} \mathrm{~cm}^{-3}$.

a period $\lambda_{b}$ and a strength parameter $K$ given by $K=\gamma \theta$ $=\gamma k_{b} r_{0}=1.33 \times 10^{-10} \sqrt{\gamma n_{e}\left(\mathrm{~cm}^{-3}\right)} r_{0}(\mu \mathrm{m})$, where $\theta$ is the peak angular deflection of the electron trajectory (Fig. 1). Consequently, as in a conventional insertion device, synchrotron radiation is emitted by the relativistic electron. The electron trajectory determines the divergence of the produced radiation. It consists of a narrow cone of divergence $\theta=K / \gamma \mathrm{di}$ rected in the forward direction. The radiation is emitted in regimes that are distinguished by the strength parameter $K$. For $K \ll 1$, the electron motion is near axis and has a weak amplitude. The ion cavity then acts as an undulator and the radiation is primarily emitted at the fundamental frequency

$$
\omega_{f}=\omega_{b}\left(2 \gamma^{2}\right)=(2 / \sqrt{2}) \omega_{p} \gamma^{3 / 2},
$$

which corresponds to the betatron frequency Doppler shifted in the laboratory reference frame. For $K>1$, the amplitude of the electron motion is increased, the plasma acts as a wiggler, high harmonics are radiated, and broadband radiation is produced. In that case, the spectrum is described by the function $S\left(\omega / \omega_{c}\right)=\left(\omega / \omega_{c}\right) \int_{\omega / \omega_{c}}^{\infty} K_{5 / 3}(x) d x$, characteristic of the synchrotron radiation. ${ }^{11}$ The spectral intensity grows up to a frequency given by

$$
\omega_{c}=(3 / 2) \gamma^{3} c / R=(3 / 2) \gamma^{3} c r_{0} k_{b}^{2},
$$

where $R=1 /\left(r_{0} k_{b}^{2}\right)$ is the effective radius of curvature of the electron path, and then exponentially decreases. We have calculated the radiation emitted by the electron oscillating in the ion channel by using the trajectories obtained above. The general expression for the spectral flux emitted in the direction of observation $\vec{n}$ is given by ${ }^{11}$

$$
\frac{d^{2} I}{d \omega d \Omega}=\frac{e^{2}}{4 \pi^{2} c}\left|\int_{-\infty}^{+\infty} e^{i \omega[t-\vec{n} \cdot \vec{r}(t) / c]} \frac{\vec{n} \times[(\vec{n}-\vec{\beta}) \times \dot{\vec{\beta}}]}{(1-\vec{\beta} \cdot \vec{n})^{2}} d t\right|^{2},
$$

where $\vec{r}(t), \vec{\beta}(t)$, and $\dot{\vec{\beta}}(t)$ are, respectively, the position, the normalized velocity, and the acceleration of the electron as a function of time (obtained previously from the electron equa- 

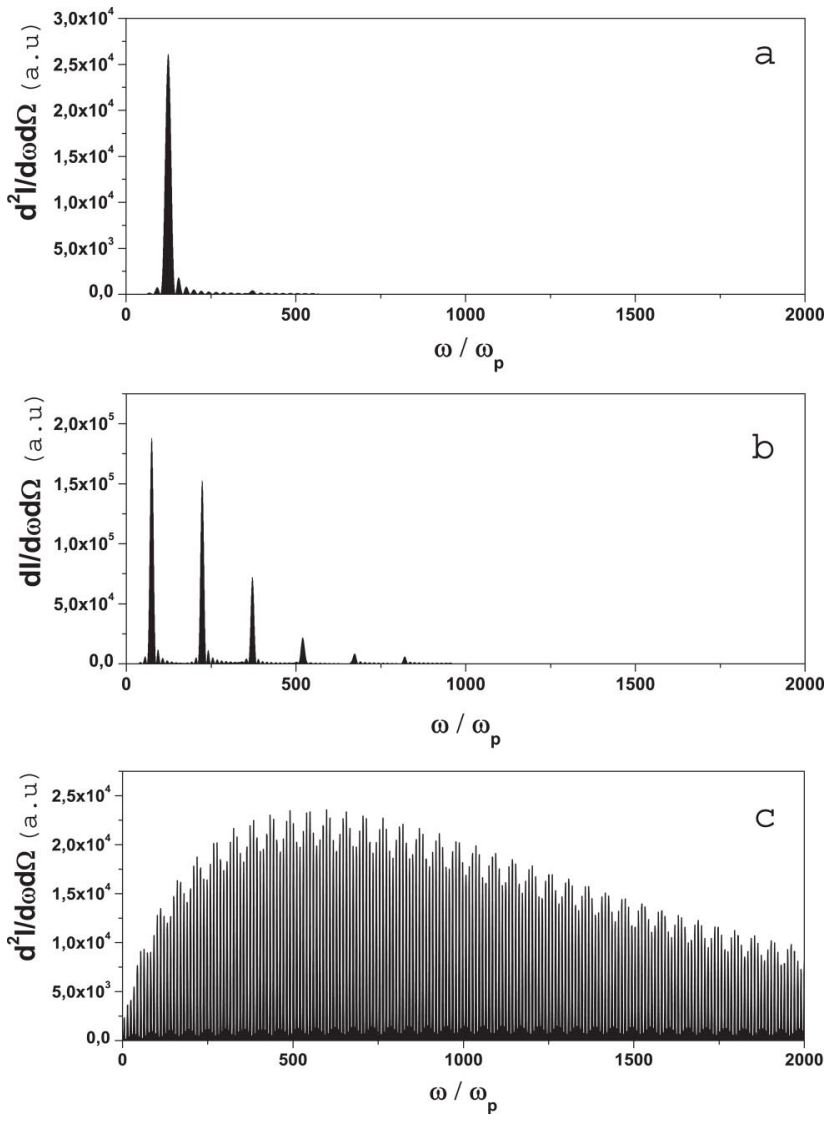

FIG. 3. Spectra on axis for (a) $\left(\gamma_{0}=20, r_{0}=0.1 \mu \mathrm{m}\right) K=0.18$, (b) $\left(\gamma_{0}=20\right.$, $\left.r_{0}=0.5 \mu \mathrm{m}\right) K=0.94$, and (c) $\left(\gamma_{0}=20, r_{0}=3 \mu \mathrm{m}\right) K=5.6$. The plasma density is $n_{e}=1 \times 10^{19} \mathrm{~cm}^{-3}$. For $K \ll 1$, the radiation is emitted at the fundamental. As the amplitude of the betatron oscillation increases, the electron radiates harmonics. For $K \gg 1$, the spectrum is a broadband continuum.

tion of motion). The spectrum of the radiation estimated on the laser axis (in the propagation direction) is presented in Fig. 3 for the sets of parameters $\left(\gamma=20, r_{0}=0.01\right) K=0.018$, $\left(\gamma=20, r_{0}=0.1\right) K=0.18$, and $\left(\gamma=20, r_{0}=3\right) K=5.6$. For $K$ $=0.018$ and $\gamma=20$, the radiation is emitted in the undulator regime at the fundamental frequency: $\omega / \omega_{p}=(2 / \sqrt{2}) \gamma^{3 / 2}$ $=126.5$. For $K \sim 1$, the first few harmonics appear. For $K$ $=5.6$, the radiation is emitted in the wiggler regime and has a broadband spectrum peaked at $\omega_{c} / \omega_{p}=(3 / 4 c) \gamma^{2} \omega_{p} r_{0}=540$. In this paper we will consider electrons accelerated in a plasma by wakefield with energies extending up to 200 $\mathrm{MeV}^{5}$ As a first example, let us consider an electron with $\gamma=60$ and with an amplitude of oscillation $r_{0}=5 \mu \mathrm{m}$. Figure 4 represents the spectrum obtained in that case for which $K$ $=16$. It extends from $\mathrm{eV}$ to a few tens of $\mathrm{keV}$ and is peaked at $1 \mathrm{keV}$. The radiation is collimated in the forward direction with a divergence $(\theta=K / \gamma)$ of a few hundreds mrad and maximum on axis. Finally, the average number of photons with mean energy $\hbar \omega_{c}$ emitted by one electron is given by Ref. $7, N_{x}=5.6 \times 10^{-3} N_{0} K$, where $N_{0}$ is the number of betatron oscillations undergone by the electron. For $\gamma=60, n_{e}$ $=10^{19} \mathrm{~cm}^{-3}$, and an amplitude of oscillation $r_{0}=5 \mu \mathrm{m}$ (obtained from PIC simulation), an $\mathrm{x}$-ray beam providing 8 $\times 10^{-2}$ photons/electron/betatron oscillation centered at an energy around $1 \mathrm{keV}$ within a full divergence of $300 \mathrm{mrad}$

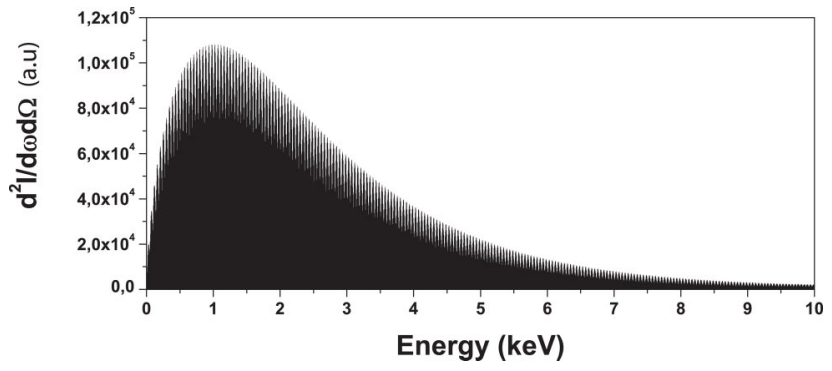

FIG. 4. Spectrum estimated on axis for an electron with $\left(\gamma_{0}=60, r_{0}\right.$ $=5 \mu \mathrm{m})$. The plasma density is $n_{e}=1 \times 10^{19} \mathrm{~cm}^{-3}$. The wiggler strength parameter is $K=16$.

could be produced. For properly chosen parameters, we therefore expect to generate an intense beam of $\mathrm{keV}$ radiation during the relativistic laser-plasma interaction.

\section{X-RAY FEATURES FROM 3D PIC SIMULATIONS}

Electrons accelerated in plasmas by wakefields have a broad energy spectrum and their trajectories in the plasma are much more complex than in the simple model depicted in the preceding section. To obtain a more accurate description of the laser plasma interaction in this high intensity regime, which comprises the electron acceleration, the ion channel formation and the resulting $\mathrm{x}$-ray radiation, we have used a 3D PIC code ${ }^{12}$ that has been modified to properly model the synchrotron emission. In the code, we suppose that at any given moment of time, the relativistic electron emits, along its momentum direction, a radiation spectrum defined by the function $S\left(\omega / \omega_{c}\right)$. We follow the trajectories of each electron and calculate the emission during the interaction. The emitted radiation, exerting a recoil on the electron, ${ }^{11}$ is included into the equations of the electron motion. In the calculation we have used the parameters of the experiment. The laser pulse is Gaussian, $a(t, r)=a_{0} \exp \left(-r_{\perp}^{2} / r_{L}^{2}-t^{2} / T_{L}^{2}\right)$, with a wavelength $\lambda=0.820 \mu \mathrm{m}$, where $a=e A / m c^{2}$ is the normalized vector potential, $r_{L}=18 \mu \mathrm{m}, T_{L}=30 \mathrm{fs}$, and an initial $a_{0}=1.2$. For these parameters, the laser power exceeds the critical power for relativistic self-focusing. The PIC simulation shows that the laser beam self-focuses, and $a_{0}$ is slightly increased in the plasma. As the laser propagates in the plasma, it leaves a large amplitude plasma wave in which electrons are trapped and accelerated up to about $100 \mathrm{MeV}$ and an ion channel almost free of background electrons. ${ }^{6,7}$ In the channel, the simulation shows that the electrons execute betatron oscillations with an amplitude of a few microns and produce a beam of $\mathrm{keV} x$ rays. The maximum $\mathrm{x}$-ray emission is observed at $n_{e}=1 \times 10^{19} \mathrm{~cm}^{-3}$. At this density, the wake amplitude maximizes because the laser pulse duration resonantly fits the relativistically corrected plasma wave period $T_{p}=2 \pi / \omega_{p}$, where $\omega_{p}^{2}=4 \pi n_{e} e^{2} /(\gamma m)$. The x-ray spectrum obtained numerically, represented as a function of the observation angle, for the optimal plasma density $n_{e}=1$ $\times 10^{19} \mathrm{~cm}^{-3}$, and for a laser propagation distance of $3 \mathrm{~mm}$, is shown in Fig. 5. It gives the number of X-ray photons emitted within $0.1 \%$ of the energy bandwidth $\left(\Delta \hbar \omega=10^{-3} \hbar \omega\right)$ per solid angle $(2 \pi \sin \theta d \theta)$. The energy and the angle $\theta$ axes are logarithmically scaled. In our parameter regime, the ex- 


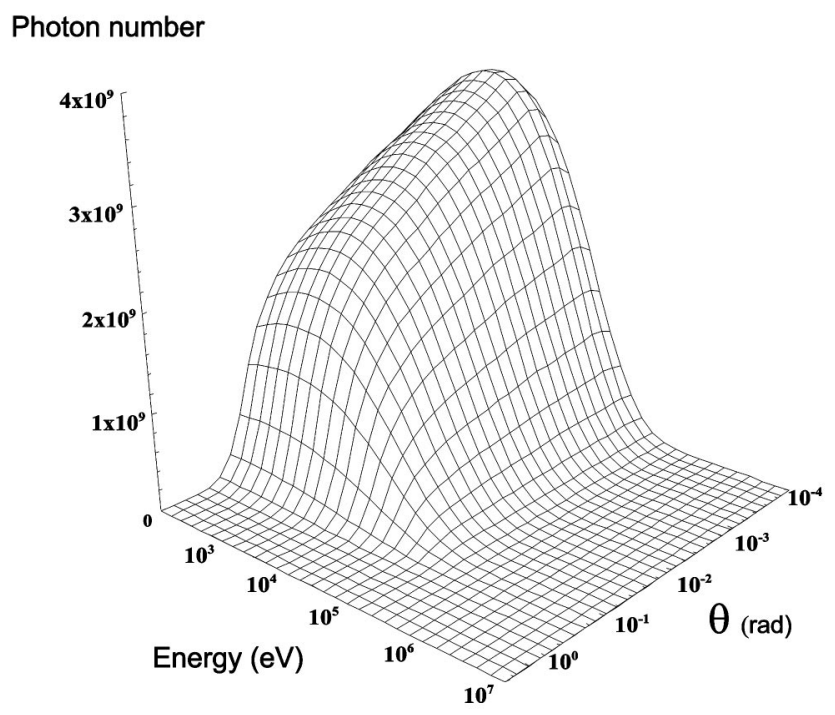

FIG. 5. The numerically predicted synchrotron spectrum from the plasma with density $n_{e}=1 \times 10^{19} \mathrm{~W} / \mathrm{cm}^{2}$ after $3 \mathrm{~mm}$ propagation distance. The distribution gives the number of $\mathrm{x}$-ray photons emitted within $0.1 \%$ of the energy bandwidth $\left(\Delta \hbar \omega=10^{-3} \hbar \omega\right)$ per solid angle, $2 \pi \sin \theta d \theta$.

pected spectrum is a continuum with a maximum of the $\mathrm{x}$-ray emission located in the energy range of $1 \mathrm{keV}$, and the divergence of the X-ray beam is about $50 \mathrm{mrad}$ full width at half maximum (FWHM). This is in close agreement with the simple test-particle model described previously.

\section{EXPERIMENTAL SETUP}

The experiment was performed at the Laboratoire d'Optique Appliquée using a titanium-doped sapphire (Ti:Sa) laser operating at $10 \mathrm{~Hz}$ with a wavelength $\lambda_{0}$ of $820 \mathrm{~nm}$ in chirped-pulse amplification mode. ${ }^{13}$ The laser delivers energies up to $1 \mathrm{~J}$ on target in $30 \mathrm{fs}$ (FWHM) pulses, with a linear horizontal polarization. The laser beam was focused with an $f / 18$ off-axis parabolic mirror onto the edge of a supersonic helium gas jet (diameter $3 \mathrm{~mm}$ ), which was characterized by interferometry. The density profile shows high uniformity throughout the jet as well as sharp edges. ${ }^{14}$ The laser distribution at full energy in the focal plane was Gaussian with a waist $w_{0}$ of $18 \mu \mathrm{m}$ containing $50 \%$ of the total laser energy. This produces vacuum-focused intensities $I_{L}$ on the order of $3 \times 10^{18} \mathrm{~W} / \mathrm{cm}^{2}$ for which the corresponding normalized vector potential $a_{0}$ is 1.2 . By varying the backing pressure of the gas jet, the electron plasma density can be tuned between $2 \times 10^{18} \mathrm{~cm}^{-3}$ and $6 \times 10^{19} \mathrm{~cm}^{-3}$. We followed the laser propagation in the plasma using side and top Thomson scattering imaging as well as time resolved shadowgraphy. In this experiment, we characterized the electrons accelerated above a few $\mathrm{MeV}$ and $\mathrm{x}$-ray emitted in the forward direction.

\section{EXPERIMENTAL RESULTS}

At first, the electron beam created and accelerated in the plasma was characterized on axis using a magnetic electron spectrometer covering energies up to $200 \mathrm{MeV}$ and an integrating current transformer (ICT) (Fig. 6). The plasma and laser parameters were chosen to accelerate electrons in the
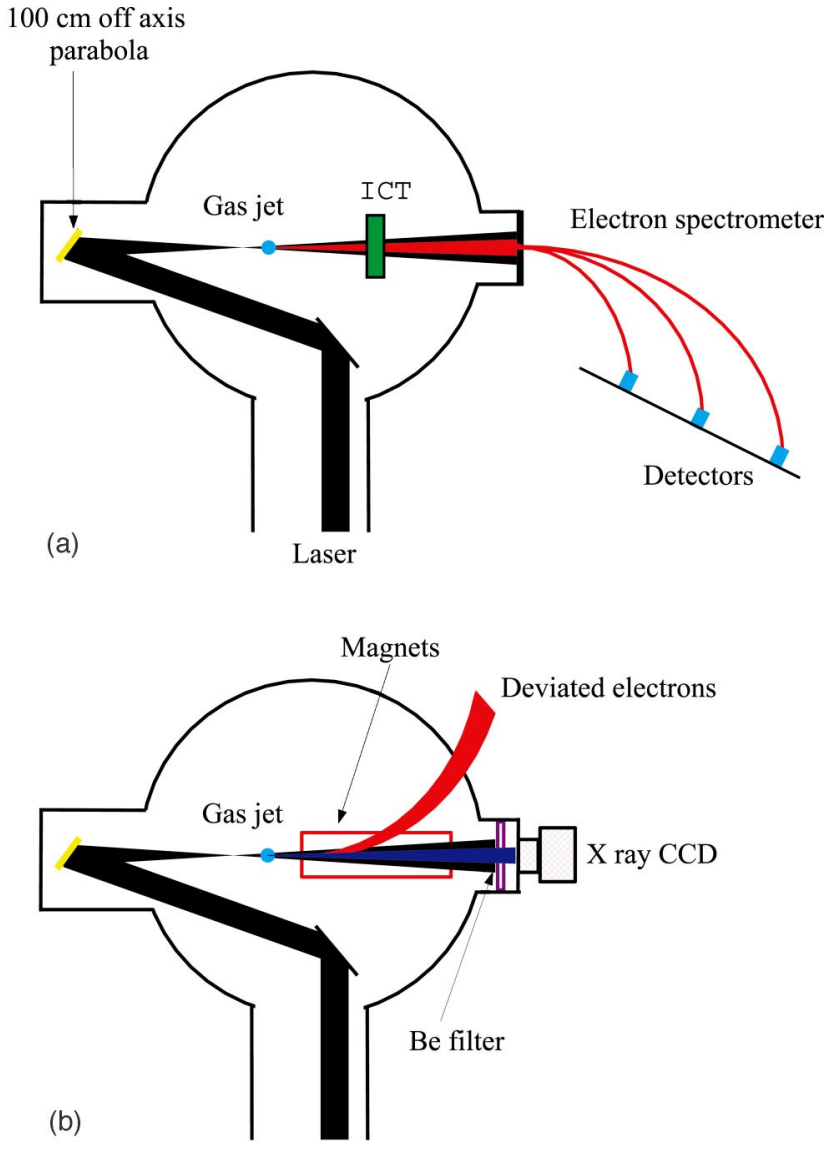

FIG. 6. (Color online). Experimental setup for the electron (a) and x-ray (b) beams characterizations.

regime of forced laser wakefield described in Ref. 5. In Fig. 7 is represented the electron spectrum measured at a plasma density $n_{e}=1 \times 10^{19} \mathrm{~cm}^{-3}$ as well as the result of $3 \mathrm{D}$ PIC simulation performed under our experimental conditions. For these parameters, electrons were detected up to $100 \mathrm{MeV}$ with an effective longitudinal temperature of $20 \mathrm{MeV}$. The total charge of the electron bunch, measured with the ICT, was about $5 \mathrm{nC}$.

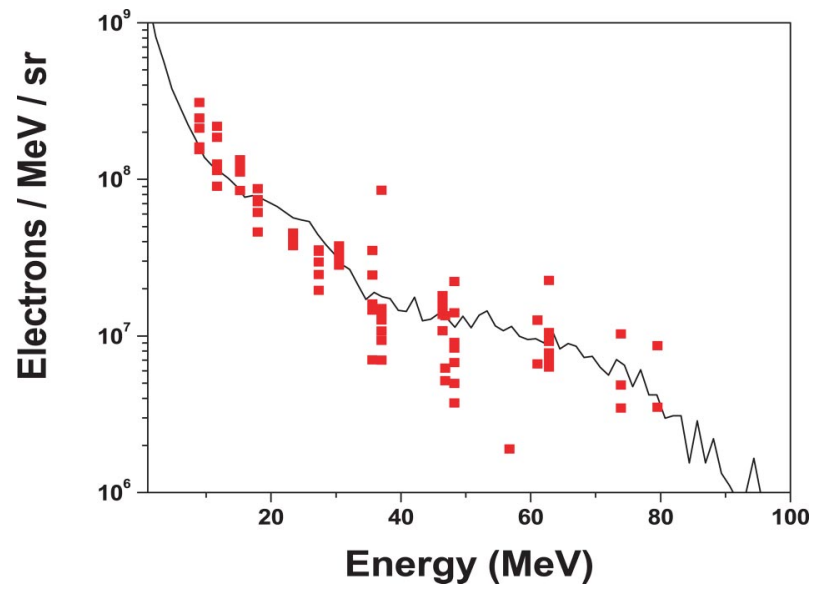

FIG. 7. (Color online). Experimental (square) electron spectrum and numerical electron spectrum (solid line) obtained using a 3D PIC simulation. 


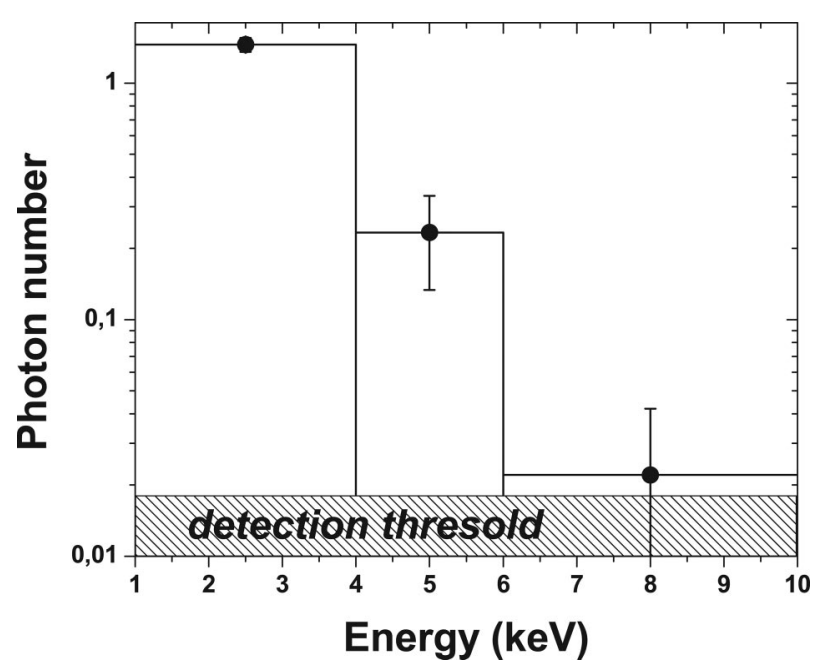

FIG. 8. X-ray flux obtained experimentally within the spectral bandwidths determined by $25 \mu \mathrm{m}$ Be filter $(1<E<10 \mathrm{keV}), 25 \mu \mathrm{m}$ Be filter $+40 \mu \mathrm{m} \mathrm{Al}$ filter $(4<E<10 \mathrm{keV}), 25 \mu \mathrm{m}$ Be filter $+25 \mu \mathrm{m} \mathrm{Cu}$ filter $(6<E<10 \mathrm{keV})$. The distribution gives the number of $\mathrm{x}$-ray photons emitted within $0.1 \%$ of the energy bandwidth and per solid angle $\left(\times 10^{8}\right)$.

The x-ray radiation produced in the plasma was measured using a cooled x-ray charge-coupled device (CCD) camera placed (50 cm after the plasma) directly on the laser axis without any focusing x-ray optic (Fig. 6). For all measurements, a $25 \mu \mathrm{m}$ beryllium filter is kept in front of the CCD camera to block any radiation below $1 \mathrm{keV}$. Permanent magnets $(0.3 \mathrm{~T}$ along $20 \mathrm{~cm})$ are inserted between the plasma (10 cm after the plasma) and the x-ray CCD to deviate offaxis the accelerated charged particles, spatially separate them from the $\mathrm{x}$ rays, and minimize the noise level onto the detector. We have characterized the x-ray emission by measuring its spectral and spatial distribution, the x-ray intensity and the spatial distribution as a function of the electron density of the plasma, the variation of the flux with the laser energy. We have observed a beam of x-ray radiation. The radiation is intense, broadband in the $\mathrm{keV}$ spectral range, and confined in the forward direction within a $50 \mathrm{mrad}$ cone (FWHM). As we will see, the properties of this X-ray beam are in a good agreement with the synchrotron radiation emitted by the trapped electrons undergoing betatron oscillations in an ion channel as it is described by the numerical simulations.

The spectral distribution of the radiation was measured from $1 \mathrm{keV}$ to $10 \mathrm{keV}$ by placing a set of $\mathrm{Be}, \mathrm{Al}, \mathrm{Cu}$, and $\mathrm{Ni}$ filters in front of the detector. The back-illuminated CCD did not allow the characterization of the radiation above $10 \mathrm{keV}$ due to the weak sensitivity for high $\mathrm{x}$-ray energies. Furthermore, the spectral resolution was limited by the bandwidths of the filters. We can see in Fig. 8 that the spectrum decreases exponentially from 1 to $10 \mathrm{keV}$. The total number of photons (integrated over the bandwidths of the filters and over the divergence of the $\mathrm{x}$-ray beam) is found to be more than $10^{8}$ photons (per shot/solid angle at $0.1 \% \mathrm{BW}$ ). The spectrum observed is in reasonable agreement with the testparticle and PIC simulations, even if slight differences are observed in the shape and the total photons number. This is not surprising if we consider the large fluctuations of the
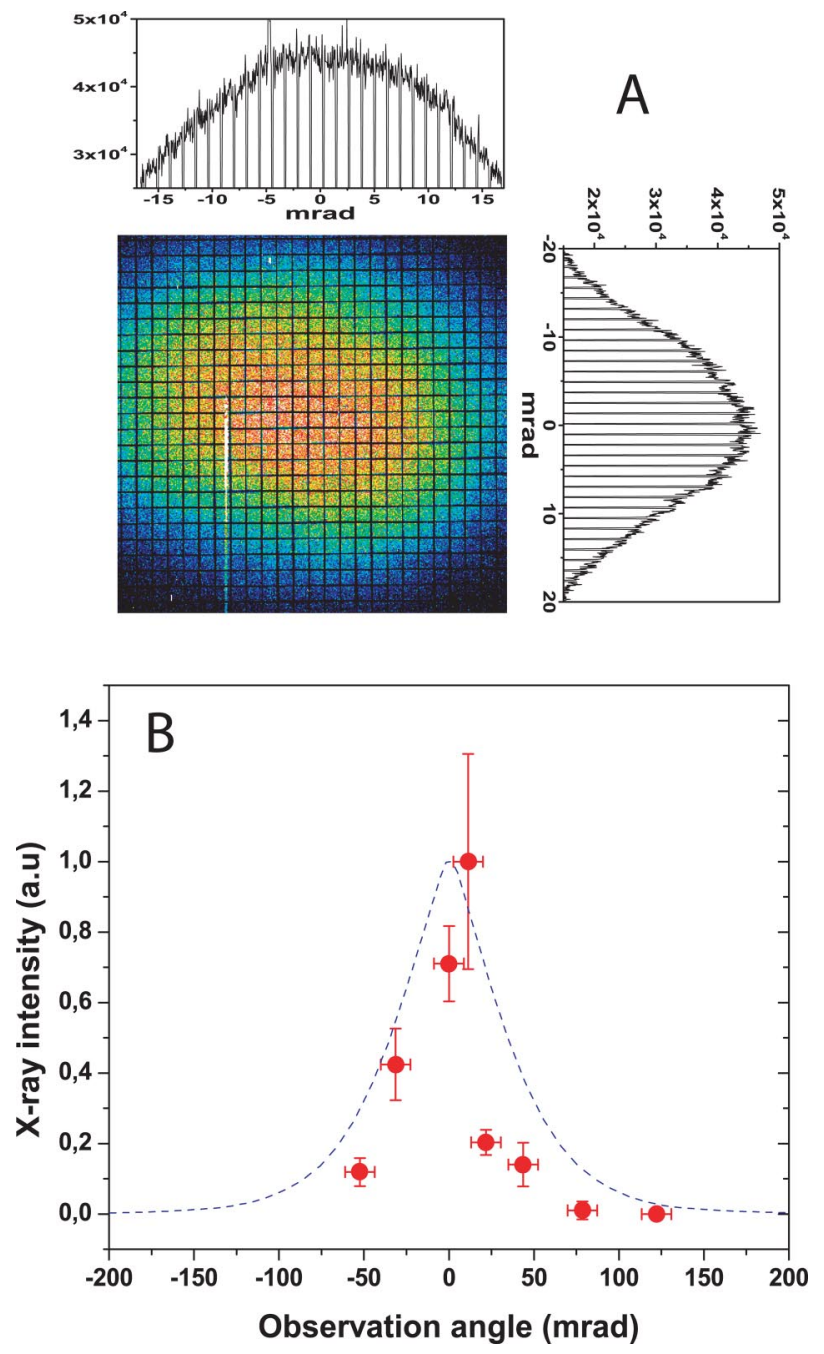

FIG. 9. (Color online). Angular distribution of the radiation for x-ray integrated energies beyond $1 \mathrm{keV}$. (a) Beam profile of the x-ray beam at $n_{e}=8$ $\times 10^{18} \mathrm{~cm}^{-3}$ and the corresponding lineout graphics. (b) Angular distribution of the x-ray intensity for $n_{e}=1.1 \times 10^{19} \mathrm{~cm}^{-3}$ (dotted line). The x-ray intensity has been normalized.

electron spectrum as shown in Fig. 7 and the dependence of the betatron x-ray radiation on the electron parameters $\left(\omega_{c} \alpha \gamma^{2}, N_{X} \alpha \sqrt{\gamma}, \theta \alpha 1 / \sqrt{\gamma}\right)$. More statistics, simultaneous measurements of the electron and $\mathrm{x}$-ray spectrum and spatial divergence as a function of the $\mathrm{x}$-ray energy are needed to allow a reliable and fine comparison between the PIC simulation and the experimental results.

The most important feature of the observed x-ray emission is its collimation in a low divergence beam centered onto the laser axis. As shown on the beam profile in Fig. 9, recorded for x-ray energies above $1 \mathrm{keV}$ and for $n_{e}=8$ $\times 10^{18} \mathrm{~cm}^{-3}$, the beam divergence is found to be $20 \mathrm{mrad}$ (FWHM). In addition, we observed that it strongly depends on the electron density and goes from $20 \mathrm{mrad}$ to $50 \mathrm{mrad}$ (FWHM) as $n_{e}$ is increased to $1.1 \times 10^{19} \mathrm{~cm}^{-3}$. At larger densities, the $\mathrm{x}$-ray beam divergence is further increased. This feature can be correlated to the $K$ parameter, proportional to the divergence $(\theta=K / \gamma)$, which increases as $n_{e}$ is increased. It is also strongly linked to the divergence of the electron beam for which similar dependencies are observed. For $n_{e}$ 


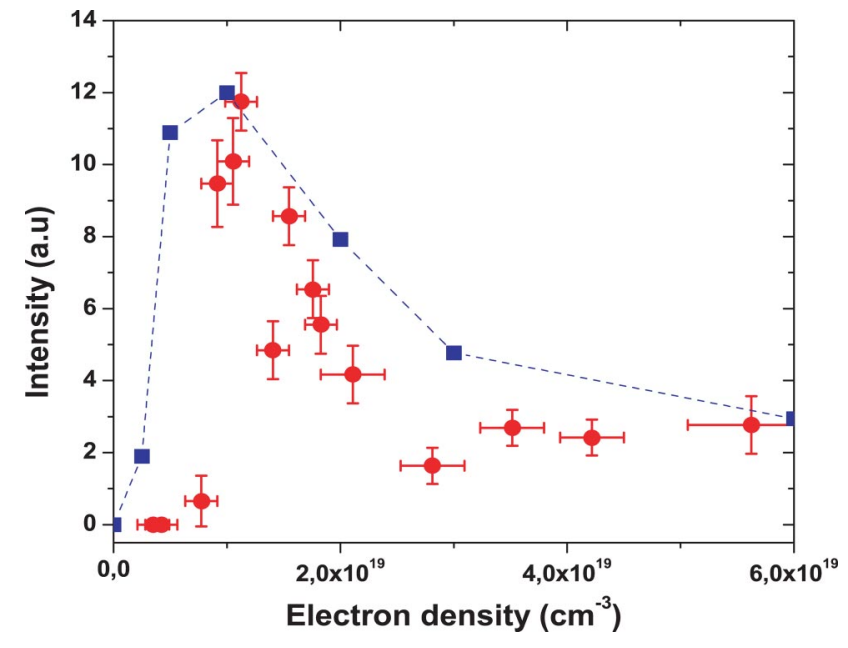

FIG. 10. (Color online). X-ray signal as a function of the plasma electron density for $\mathrm{x}$-ray energies beyond $1 \mathrm{keV}$. Each data point corresponds to an average value over ten shots. The square along the dotted line corresponds to the results obtained using a 3D PIC simulation.

$=1.1 \times 10^{19} \mathrm{~cm}^{-3}$, at which the $\mathrm{x}$-ray intensity is maximum, the spatial distribution was larger than the size of the CCD area (using our experimental setup). These results were then obtained by rotating the x-ray CCD around the gas jet. The $\mathrm{x}$-ray beam divergence, averaged over more than ten shots, is found to be $\Delta \theta=50 \pm 20 \mathrm{mrad}$ at FWHM.

Another unique feature of the x-ray beam is its intensity as a function of the electron density of the plasma. It is found to be sharply peaked at $n_{e}=1.1 \times 10^{19} \mathrm{~cm}^{-3}$ (Fig. 10). Below this critical density, the $\mathrm{x}$-ray signal rapidly vanishes mainly because the number of trapped electrons is too low. This is confirmed in the experiment for which no electrons were detected by the spectrometer. At larger densities the $\mathrm{x}$-ray signal drops down and a plateau is reached. For these experimental conditions, the resulting amplitude of the plasma wave becomes too weak. The pulse must first be modulated and additional laser energy would be needed. As a result, the temperature of the electron beam decreases and its divergence increases. The PIC simulations clearly reproduce this experimental behavior: a sharp increase of the $\mathrm{x}$-ray intensity followed by a smoother decrease of the signal.

We have determined the radiation source size in the transverse direction by using shadows by the wires of the nickel grid acting as knife edges. As shown in Fig. 11, the grid was placed along the $\mathrm{x}$-ray beam path at $10 \mathrm{~cm}$ from the source. The detector was located $40 \mathrm{~cm}$ after the grid where its shadow is detected. A simple geometrical relation then gives a transverse source size $(20 \mu \mathrm{m} \times 20 \mu \mathrm{m})$ from the shadow of any wire of the mesh. It is in the same order of magnitude than the laser focal spot.

Other radiative processes, such as relativistic Bremsstrahlung radiation or nonlinear Thomson scattering, could potentially produce a collimated beam of $\mathrm{x}$ rays in the $\mathrm{keV}$ energy range. However, the intensity of the relativistic bremsstrahlung radiation-emitted by the electrons undergoing collisions with the ionic background-is too inefficient under our experimental conditions to explain the results. Much higher electron density and laser intensity would be

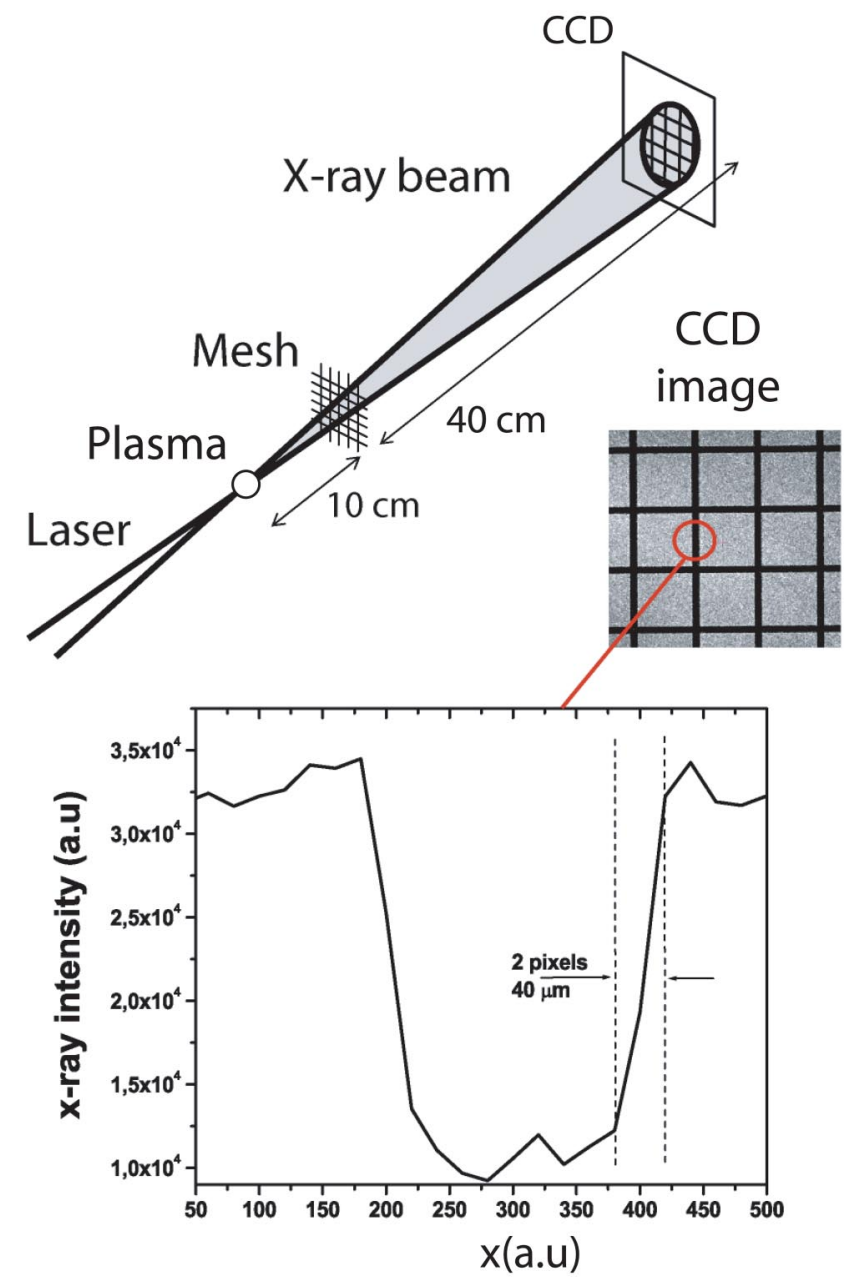

FIG. 11. (Color online). Schematic diagram of the source size measurement. We placed a $13 \mu \mathrm{m}$ diameter wire mesh in the beam. The shadow of the mesh obtained on the detector is shown on the lower figure. A simple geometrical relation provides the source size in the transverse direction from the line-out graph presented here (knife edge technique).

required. Nonlinear Thomson scattering radiation, emitted by the electrons of the plasma oscillating in the intense laser field, has been proposed as a source of femtosecond x rays, ${ }^{15}$ and was demonstrated, in a collinear geometry, to produce high-order harmonics in the vacuum ultraviolet (vuv) range, ${ }^{16}$ and continuum radiation in the $100 \mathrm{eV}$ range (for $\left.a_{0} \sim 6\right) .{ }^{17}$ But, in this geometry, much higher laser intensity (at least $a_{0} \sim 10$ ) than used in the present experiment would be needed to extend the spectrum up to the $\mathrm{keV}$ range.

\section{PERSPECTIVES}

We have measured the betatron radiation of relativistic electrons oscillating in an ion channel. The features of the observed radiation are in agreement with the analytical estimations and the numerical simulations. According to the theory, we have also observed that the x-ray and electron beams properties are closely linked.

The x-ray flux as a function of the laser energy is presented in Fig. 12. A minimum laser energy is needed to observe the betatron emission. This energy threshold is related to the wave breaking condition to accelerate background 


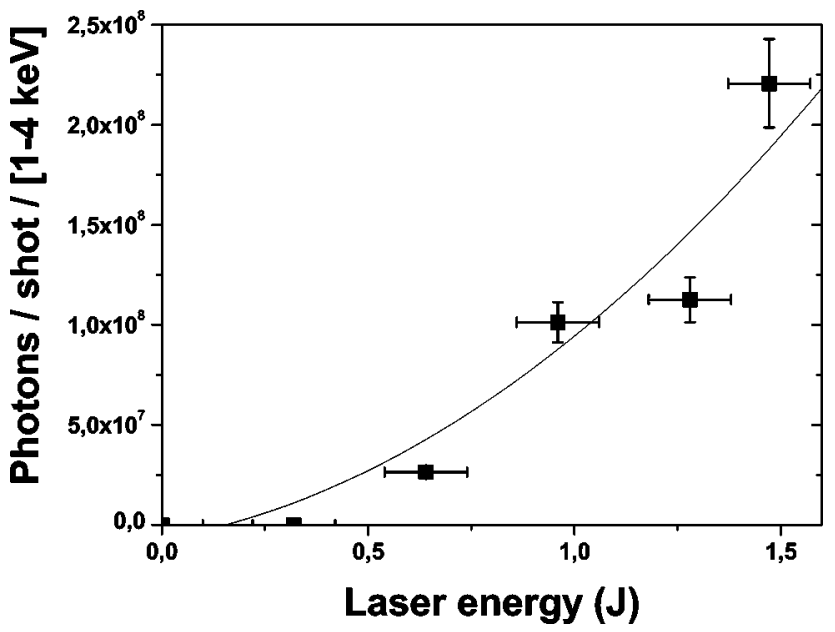

FIG. 12. X-ray signal as a function of the laser energy.

electrons. Below this energy, no accelerated electrons are observed, while above, the total x-ray flux increases nearly as the square of the laser energy. In addition, as it was already mentioned, an increase of the $\gamma$ factor of the electron result in a significant increase of the x-ray flux, energy, and produces a more collimated beam. The energy of the electron beam can be enhanced by increasing the laser pulse energy. In the highly nonlinear broken wave regime, ${ }^{6}$ numerical simulations have shown that monoenergetic electron beams can be produced at about $300 \mathrm{MeV}$ by using a $33 \mathrm{fs}, 12 \mathrm{~J}$ (363 TW) laser. The number of electrons in the bunch is about $3 \times 10^{10}$. In this regime, the maximum electron energy scales roughly like $a_{0}$. Recently, monoenergetic beams of electrons up to $180 \pm 20 \mathrm{MeV}$ have already been produced experimentally at LOA using a $100 \mathrm{TW}$ laser. ${ }^{18}$

Following these results, it is realistic to consider that electrons will be accelerated up to $300 \mathrm{MeV}$ in a near future. Assuming that these electrons undergo betatron oscillations with an amplitude $r_{0}=5 \mu \mathrm{m}$ in a plasma at the density $n_{e}$ $=10^{19} \mathrm{~cm}^{-3}$, the plasma wiggler strength parameter is then $K=1.33 \times 10^{-10} \sqrt{\gamma n_{e}} r_{0} \sim 50$. Because $K \gg 1$, the produced radiation has a broad synchrotronlike spectrum and the maximum x-ray energy produced by the electrons beam is expected at $0.29 \hbar \omega_{c} \sim 26 \mathrm{keV}$. The Fig. 13 displays the result of the test-particle simulation performed under these conditions. We have also represented the spectrum for electrons with $\gamma=400$ and $\gamma=200$. As we can see the peak of the spectrum shifts to high energy as the electron energy is increased and $\mathrm{x}$-ray radiation at about 5,10 , and $30 \mathrm{keV}$ is expected to be produced for respectively $\gamma=200,400$, and 600.

The number of photons emitted by one electron, over one betatron oscillation, is given by $N_{X}=5.6 \times 10^{-3} N_{0} K \sim 1$ (for $N_{0}=1$ ). If we consider that the electron executes five betatron oscillations (for these parameters $\lambda_{b} \sim 360 \mu \mathrm{m}$ ) 5 $\times 10^{10} \mathrm{x}$-ray photons are produced for $1 \times 10^{10}$ electrons in the bunch. Finally, the total divergence of the beam is $\theta$ $=K / \gamma=80 \mathrm{mrad}$.

By using a grazing incidence mirror, all x-ray photons with energies below about $10 \mathrm{keV}$ (for reflectivity reasons)

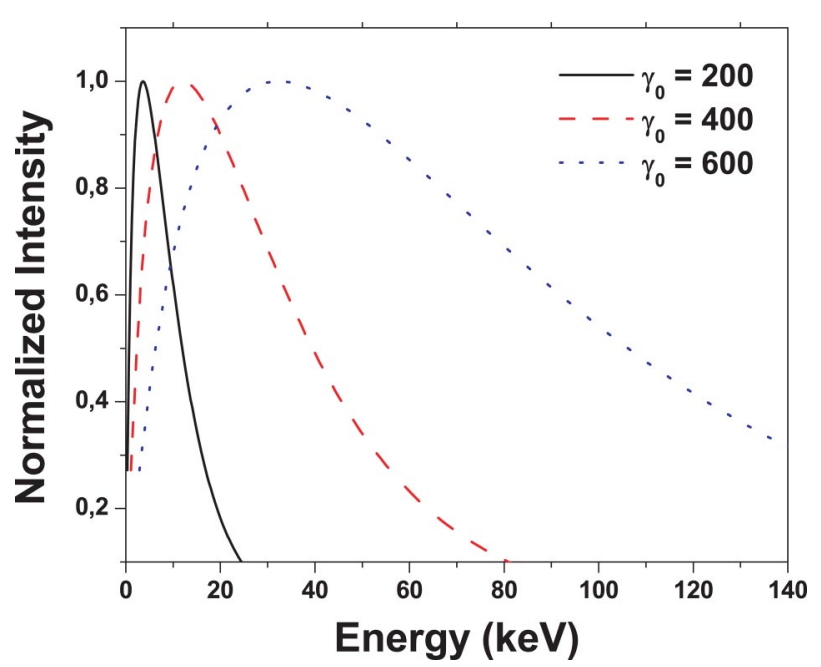

FIG. 13. (Color online). Spectrum of the betatron radiation for electrons energies $\gamma=600$ (dotted line), $\gamma=400$ (dashed line), and $\gamma=200$ (solid line). In all the cases the intensity is normalized.

can be collected and focused. On target, intensities as high as $10^{12} \mathrm{~W} / \mathrm{cm}^{2}$ can be achieved which corresponds to about $10^{9}$ photons focussed on a $30 \mu \mathrm{m}$ focal spot. In $0.1 \%$ of the bandwidth it corresponds to $10^{6}$ photons at $4 \mathrm{keV}$ in the beam. It is two orders of magnitude more than the $K \alpha$ source.

\section{CONCLUSION}

The $\mathrm{x}$-ray radiation produced by relativistic electrons undergoing betatron oscillations in ion channel combines synchrotron features and femtosecond pulse duration in a compact source. We have observed a collimated beam of $\mathrm{keV}$ radiation with a broad energy spectrum. The x-ray pulse duration has not yet been measured but should be of the same order of the duration of the electron bunch which is expected to be femtosecond. ${ }^{5,6}$ The brightness of the produced $\mathrm{x}$-ray radiation is estimated from the pulse duration and the size of the $\mathrm{x}$-ray source. The average brightness is 5 $\times 10^{6} \mathrm{ph} / \mathrm{s} / \mathrm{mm}^{-2} / \mathrm{mrad}^{-2} / 0.1 \% \mathrm{BW}$ and the peak spectral brightness is $2 \times 10^{22} \mathrm{ph} / \mathrm{s} / \mathrm{mm}^{-2} / \mathrm{mrad}^{-2} / 0.1 \% \mathrm{BW}$.

Beyond the demonstration that compact synchrotron radiation can be produced with femtosecond laser systems, this radiative process opens very promising perspectives in a near future. Presently, its reasonably high flux (5 $\times 10^{6}$ photons/pulse/s $\left./ 0.1 \% \mathrm{BW}\right)$ and its perfect synchronization with the laser system enables visible pump/x-ray probe experiments with insignificant time jitter. MultipleBragg diffraction and absorption x-ray studies can be achieved, which will significantly extend the first x-ray diffraction studies already demonstrated in ultrafast $\mathrm{x}$-ray science. $^{19-22}$

\section{ACKNOWLEDGMENTS}

This work was supported by the European Community under Contract Nos. HPRI-CT-1999-00086, HPRI-CT-200040016, and HPRI-CT-1999-50004 (FAMTO project). K.T.P received support from the U.S. Nat. Sci. Fond. FOCUS cen- 
ter. D.U. and R.S. were supported by Department of Energy, Chemical Sciences, Geosciences and Biosciences Divisions, Office of Science.

${ }^{1}$ A. Rousse, K. T. Phuoc, R. Shah, A. Pukhov, E. Lefebvre, V. Malka, S. Kiselev, F. Burgy, J.-P. Rousseau, D. Umstadter, and D. Hulin, Phys. Rev. Lett. 93, 135005 (2004).

${ }^{2}$ A. Tarasevitch, A. Orisch, D. von der Linde, Ph. Balcou, G. Rey, J.-P. Chambaret, U. Teubner, D. Klpfel, and W. Theobald, Phys. Rev. A 62, 023816 (2000); H. Daido, Rep. Prog. Phys. 65, 1513 (2002); A. Th. Brabec and F. Krausz, Rev. Mod. Phys. 72, 545 (2000).

${ }^{3}$ A. Rousse, P. Audebert, J. P. Geindre, F. Fallis, J. C. Gauthier, A. Mysyrowicz, G. Grillon, and A. Antonetti, Phys. Rev. E 50, 2200 (1994); M. Murnane et al., Science 251, 531 (1991); J. C. Kieffer et al., Phys. Fluids B 5, 2676 (1993).

${ }^{4}$ A. Rousse, C. Rischel, and J. C. Gauthier, Rev. Mod. Phys. 73, 17 (2001).

${ }^{5}$ V. Malka, S. Fritzler, E. Lefebvre, M.-M. Aleonard, F. Burgy, J.-P. Chambaret, J.-F. Chemin, K. Krushelnick, G. Malka, S. P. D. Mangles, Z. Najmudin, M. Pittman, J.-P. Rousseau, J.-N. Scheurer, B. Walton, and A. E. Dangor, Science 298, 1596 (2002).

${ }^{6}$ A. Pukhov and J. Meyer-ter-Vehn, Appl. Phys. B: Lasers Opt. 74, 355 (2002).

${ }^{7}$ I. Kostyukov, S. Kieselev, and A. Pukhov, Phys. Plasmas 10, 4818 (2003); E. Esarey et al., Phys. Rev. E 65, 056505 (2002).

${ }^{8}$ E. Esarey, B. A. Shadwick, P. Catravas, and W. P. Leemans, Phys. Rev. E 65, 056505 (2002).

${ }^{9}$ D. H. Whittum, Phys. Fluids B 4, 730 (1992).

${ }^{10}$ The synchrotron radiation emitted from electrons undergoing betatron motion has been studied numerically and theoretically, and observed at the Stanford Linear Accelerator where 5 ps x-ray pulses were produced using
$28.5 \mathrm{GeV}$ electrons bunch sent in a $1.4 \mathrm{~m}$ long plasma. S. Wang et al., Phys. Rev. Lett. 88, 135004 (2002); C. Joshi et al., Phys. Plasmas 9, 1845 (2002).

${ }^{11}$ J. D. Jackson, Classical Electrodynamics (Wiley, New York, 1975).

${ }^{12}$ Virtual Laser Plasma Laboratory PIC code. It is discussed in Refs. 6 and 7.

${ }^{13}$ M. Pittman, S. Ferre, J. P. Rousseau, L. Notebaert, J. P. Chambaret, and G. Chriaux, Appl. Phys. B: Lasers Opt. 74, 529 (2002).

${ }^{14}$ V. Malka, C. Coulaud, J. P Geindre, V. Lopez, Z. Najmudin, D. Neely, and F. Amiranoff, Rev. Sci. Instrum. 71, 23 (2000)

${ }^{15}$ Y. Ueshima, Y. Kishimoto, A. Sasaki, T. Tajima, Laser Part. Beams 17, 45 (1999); E. Esarey, S. K. Ride, and P. Sprangle, Phys. Rev. E 48, 3003 (1993).

${ }^{16}$ S. Banerjee, A. R. Valenzuela, R. C. Shah, A. Maksimchuk, and D. Umstadter, J. Opt. Soc. Am. B 20, 182 (2003).

${ }^{17}$ K. Ta Phuoc, A. Rousse, M. Pittman, J. P. Rousseau, V. Malka, S. Fritzler, D. Umstadter, and D. Hulin, Phys. Rev. Lett. 91, 195001 (2003).

${ }^{18}$ J. Faure, Y. Glinec, A. Pukhov, S. Kieselev, S. Gordienko, E. Lefebvre, J.-P. Rousseau, F. Burgy, and V. Malka, Nature (London) 431, 541 (2004).

${ }^{19}$ C. Rischel, A. Rousse, I. Uschmann, P. A. Albouy, J. P. Geindre, P. Audebert, J. C. Gauthier, E. Frster, J. L. Martin, and A. Antonetti, Nature (London) 390, 490 (1997).

${ }^{20}$ C. W. Siders, A. Cavalleri, K. Sokolowski-Tinten, Cs. Tth, T. Guo, M. Kammler, M. H. von Hoegen, K. R. Wilson, D. von der Linde, and C. P. J. Barty, Science 286, 1340 (1999).

${ }^{21}$ A. Rousse, C. Rischel, S. Fourmaux, I. Uschmann, S. Sebban, G. Grillon, Ph. Balcou, E. Forster, J. P. Geindre, P. Audebert, J. C. Gauthier, and D. Hulin, Nature (London) 410, 65 (2001).

${ }^{22}$ K. Sokolowski-Tinten, C. Blome, J. Blums, A. Cavalleri, C. Dietrich, A. Tarasevitch, I. Uschmann, E. Foster, M. Kammler, M. H. von Hoegen, D. van der Linde, Nature (London) 422, 287 (2003). 